\title{
The Secretory Apparatus of an Ancient Eukaryote: Protein Sorting to Separate Export Pathways Occurs Before Formation of Transient Golgi-like Compartments
}

\author{
Matthias Marti*, Yajie Li ${ }^{* \ddagger}$, Elisabeth M. Schraner ${ }^{\dagger}$, Peter Wild ${ }^{\dagger}$, \\ Peter Köhler, ${ }^{*}$ and Adrian B. Hehl*§
}

\author{
*Institute of Parasitology, University of Zürich, CH-8057 Zürich, Switzerland, and 'Electron \\ Microscopy Unit, Institutes of Veterinary Anatomy and Virology, University of Zürich, CH-8057 \\ Zürich, Switzerland.
}

Submitted August 7, 2002; Revised October 23, 2002; Accepted December 9, 2003

Monitoring Editor: Howard Riezman

\begin{abstract}
Transmission of the protozoan parasite Giardia intestinalis to vertebrate hosts presupposes the encapsulation of trophozoites into an environmentally resistant and infectious cyst form. We have previously shown that cyst wall proteins were faithfully sorted to large encystation-specific vesicles (ESVs), despite the absence of a recognizable Golgi apparatus. Here, we demonstrate that sorting to a second constitutively active pathway transporting variant-specific surface proteins (VSPs) to the surface depended on the cytoplasmic VSP tail. Moreover, pulsed endoplasmic reticulum (ER) export of chimeric reporters containing functional signals for both pathways showed that protein sorting was done at or very soon after export from the ER. Correspondingly, we found that a limited number of novel transitional ER-like structures together with small transport intermediates were generated during encystation. Colocalization of transitional ER regions and early ESVs with coat protein (COP) II and of maturing ESVs with COPI and clathrin strongly suggested that ESVs form by fusion of ER-derived vesicles and subsequently undergo maturation by retrograde transport. Together, the data supported the hypothesis that in Giardia, a primordial secretory apparatus is in operation by which proteins are sorted in the early secretory pathway, and the developmentally induced ESVs carry out at least some Golgi functions.
\end{abstract}

\section{INTRODUCTION}

The diplomonad group of protozoans including Giardia intestinalis diverged very early from the eukaryotic lineage (Sogin et al., 1989), and its status as an ancient eukaryote has attracted considerable attention in recent years. In addition to several cellular, biochemical, and genomic peculiarities,

Article published online ahead of print. Mol. Biol. Cell 10.1091/ mbc.E02-08-0467. Article and publication date are at www.molbiolcell.org/cgi/doi/10.1091/mbc.E02-08-0467.

‡Present address: Department of Parasitology, Harbin Medical University, 150086, Harbin, P.R.C.

$\$$ Corresponding author. E-mail address: ahehl@vetparas.unizh.ch. Abbreviations used: BFA, brefeldin A; COP, coat protein; CWP, cyst wall protein; ESV, encystation-specific vesicle; GFP, green fluorescent protein; IFA, immunofluorescence analysis; ORF, open reading frame; $\mathrm{PV}$, peripheral vesicle; $\mathrm{mAb}$, monoclonal antibody; SAG1, surface antigen 1 (Toxoplasma gondii); tER, transitional endoplasmic reticulum; TEM, transmission electron microscopy; VSP, variant-specific surface protein.
Giardia has a very simple endomembrane system consisting of only two definable cellular compartments: nuclear envelope/endoplasmic reticulum (ER) (Soltys et al., 1996) and the lysosome-like peripheral vesicles (PVs) (Feely and Dyer, 1987). Although there has been some speculation, a morphologically recognizable and functional Golgi apparatus, the central organelle through which all exported proteins travel in eukaryotes, seems to be absent in all developmental stages. This protozoan is therefore considered to be "Golgiless," or at least lacking this organelle in the true sense (Dacks and Doolittle, 2001).

Giardia parasites proliferate as motile trophozoites in the intestine of the mammalian host. For transmission to a new host, the trophozoites must transform into an environmentally resistant form, a process that entails secretion of an extracellular matrix. Little is known about the molecular mechanisms of secretory protein transport and targeting during the life cycle of Giardia, and nothing about the mechanisms for protein sorting. Two export pathways for secretory proteins have been identified previously. One constitu- 
tively active pathway supplies the trophozoite plasma membrane with transmembrane-anchored variant-specific surface proteins (VSPs). VSPs are shed continuously by proteolytic cleavage and replaced with newly secreted protein (Papanastasiou et al., 1996). Faithful targeting of a heterologous marker protein to the plasma membrane has been achieved by fusing a reporter gene to sequences coding for a VSP transmembrane domain and cytoplasmic tail (Marti et al., 2002), but the specific signals necessary for export to the Giardia surface are not known.

The second major secretory pathway in Giardia is for export of the soluble cyst wall proteins (CWPs) and other material associated with the cyst wall, an extracellular matrix composed of at least three known CWPs (Lujan et al., 1995b; Mowatt et al., 1995) (Marti and Hehl, unpublished observations) and glycans. Synthesis and export of this material is tightly regulated and entails de novo generation of a third membrane compartment: the encystation-specific vesicles (ESVs, $\sim 1 \mu \mathrm{m}$ in diameter) (Reiner et al., 1989), which secrete their accumulated contents simultaneously, presumably in response to an internal signal. Targeting of the soluble CWPs to ESVs and ultimately to the cyst wall is dependent on signals contained in an N-terminal domain (II) and a middle domain (III) in the case of CWP1 (Hehl et al., 2000).

Because the regulated (ESV pathway) and the constitutive (VSP pathway) export pathways are active simultaneously during the 15- to 20-h encystation process (McCaffery et al., 1994), Giardia must have means to sort proteins to the plasma membrane or the cyst wall, although it lacks a Golgi apparatus. One explanation could be that this ancient eukaryote harbors a primordial protein sorting and trafficking machinery, representing a cellular state before sorting functions have been assumed by a specialized organelle. An even more intriguing idea is that during encystation, transiently active compartments may form that assume certain Golgi functions. Previous data indeed hint at such a role for ESVs, which arise during a developmental transition in the life cycle during which faithful protein sorting is of vital importance (Lujan et al., 1995a; Hehl et al., 2000).

In the initial study presented here, we addressed the question of how proteins are transported to the plasma membrane via the VSP pathway and segregated from the CWPs in the ESV pathway in encysting Giardia. Using induced expression of heterologous reporter proteins, we determined the role of VSP and CWP sorting signals during Giardia development and generated transgenic cells to study these mechanisms in a synchronized manner. We also investigated molecular and structural aspects of ER export of cyst wall material and of ESV formation and maturation. Our data strongly suggest the existence of a general protein sorting machinery very early in the Giardia secretory pathway, and substantiate the idea of ESVs as transient Golgilike organelles in this unique model organism.

\section{MATERIALS AND METHODS}

\section{Cell Culture and Transfection}

Trophozoites of Giardia strains WBC6 (American Type Culture Collection [ATCC] catalog No. 50803, Manassas, VA) and H7 (ATCC catalog No. 50581) were grown vegetatively in TYI-S-33 supplemented with $10 \%$ adult bovine serum and bovine bile. Encystation was done as described (Hehl et al., 2000) by addition of porcine bile after culture for $\sim 44 \mathrm{~h}$ without bile growth factors (preencystation period) (Boucher and Gillin, 1990).

For temperature block experiments, cells were preencysted for $44 \mathrm{~h}$ at $37^{\circ} \mathrm{C}$, and encystation medium was added prewarmed to $15^{\circ} \mathrm{C}$. Incubation was continued at that temperature until the cells were harvested by cooling on ice or until the block was relieved by warming the culture to $37^{\circ} \mathrm{C}$.

For secretion assays, $1 \times 10^{6}$ cells transformed with a plasmid containing the Ssec (or wild-type cells as a control) were encysted for 3,7 , or $15 \mathrm{~h}$, harvested by cooling and centrifugation, and resuspended in $0.5 \mathrm{ml}$ PBS supplemented with $5 \mathrm{mM}$ glucose and 5 $\mathrm{mM}$ cysteine ( $\mathrm{pH} 7.1)$. Live cells were incubated at $37^{\circ} \mathrm{C}$ for $1 \mathrm{~h}$ and harvested by cooling on ice and centrifugation. Cell pellet fractions and cleared supernatant were assayed for the presence of Ssec protein using SDS-PAGE under nonreducing conditions and Western blotting with the highly reactive monoclonal antibody $(\mathrm{mAb})$ DG52 against the SAG1 exodomain (Marti et al., 2002).

Electroporation of plasmid DNA into trophozoites and selection of stable transgenic parasites were performed as described previously (Hehl et al., 2000).

\section{Construction of Plasmid Vectors}

Expression cassettes for stable transformation of Giardia trophozoites were constructed as described previously (Hehl et al., 2000; Marti et al., 2002). All cassettes were based on a pBS KS ${ }^{-}$(Stratagene, La Jolla, CA) backbone and contained a bacterial neomycin resistance gene expressed under the control of the Giardia RAN gene promoter (Sun et al., 1998; Hehl et al., 2000) for selection of transgenic parasites with the antibiotic G418 (Sigma, St. Louis, MO). Primers used in this study are listed below and in Table 1. The SVSPct gene (Marti et al., 2002) served as a template for all chimeric genes used in this study (Figure 1). This cassette consisted of a CWP1 promoter and leader sequence fused to the SAG1 exodomain (amino acids D49 to A304; GenBank accession No. X14080) amplified from Toxoplasma gondii genomic DNA (strain RH, ATCC Nr. 50174) by PCR (NsiI-BglII fragment). The SAG1 domain was fused to a BglII-PacI fragment coding for the C-terminal 43 amino acids of the VSP-H7 protein (amino acids N515 to A557; GenBank accession No. AAA18202). The chimeric genes SVSPtm and Ssec were derived by replacing this VSP C-terminus by a shorter, 38-amino-acid, fragment lacking the codons for the cytoplasmic tail (CRGKA) or by removing the entire C-terminus, respectively. Hybrid constructs SVSPct26, SVSPct9, SVSPct44, and SVSPtm 26 were constructed by replacing the upstream region in the SVSPct cassette (XbaI-NsiI fragment) with sequences coding for additional CWP1 domains (see also Table 1). Sequences coding for the CWP1 promoter and structural domains were amplified from genomic DNA, Giardia strain WBC6, as described (Hehl et al., 2000). GVSPct26 was constructed by replacing the SAG1 exodomain with a green fluorescent protein (GFP) open reading frame (ORF). SVSPct26HA was constructed by excising the BglII-PacI fragment coding for the VSP C-terminus in SVSPct 26 and replacing it with the corresponding product amplified by PCR using primers H7-BglII-s and H7HA-PacI-as. Synthesis of oligonucleotide primers and sequence confirmation of inserts by dye termination sequencing was done by Microsynth (Balgach, Switzerland).

\section{Oligonucleotide Primers Used in this Study (5'-3' Orientation)}

The oligonucleotide primers used in this study were as follows. CWP1-s: TgaattcGCCACGCATGGGCTGTCT; CWP1-as: CgaattcTCTTGAGCTGAGCTCT; CWP-LS-as: GTatgcatGACGAGCACCTCCCTCTGA; CWPC2-as: CGatgcatATCCAGGGCGATAACGTAGT; CWP-C3-as: CGatgcatAAGGTAGGGGAGCGTC; CWP-C4-as: CGatgcatAGGCGGGGTGAGGCAGT; SAG1-PacI-as: CCttaattaaTCACGCGACACAAGCTGCGATAGA; SAG1-BglII-as: CATagatctAGCCCGGCAAACTCCAGT; 
Table 1. Designations of oligonucleotide primers used for PCR amplification of domains included in expression cassettes

\begin{tabular}{|c|c|c|c|c|c|c|}
\hline \multirow[b]{2}{*}{ Cassette } & \multicolumn{2}{|c|}{$\begin{array}{l}\text { CWP1 promoter and N- } \\
\text { terminal region }(\mathrm{XbaI}-\mathrm{Nsi})\end{array}$} & \multicolumn{2}{|c|}{ Reporter domain (NsiI-BglII) } & \multicolumn{2}{|c|}{ C-terminus (BglII-PacI) } \\
\hline & Sense & Antisense & Sense & Antisense & Sense & Antisense \\
\hline SVSPct & CWP1-s & CWP-LS-as & SAG1-Nsi1-s & SAG1-BgIII-as & H7-BgIII-s & H7-PacI-as \\
\hline SVSPtm & CWP1-s & CWP-LS-as & SAG1-Nsi1-s & SAG1-BgIII-as & H7-BgIII-s & H7tm-PacI-as \\
\hline Ssec & CWP1-s & CWP-LS-as & SAG1-Nsi1-s & & & SAG1-Pac1-as \\
\hline SVSPct26 & CWP1-s & CWP-C4-as & SAG1-Nsi1-s & SAG1-BgIII-as & H7-BgIII-s & H7-PacI-as \\
\hline SVSPct26HA & CWP1-s & CWP-C4-as & SAG1-Nsi1-s & SAG1-BgIII-as & H7-BgIII-s & H7HA-PacI-as \\
\hline SVSPct9 & CWP1-s & CWP-C2-as & SAG1-Nsi1-s & SAG1-BgIII-as & H7-BgIII-s & H7-PacI-as \\
\hline SVSPct44 & CWP1-s & CWP-C3-as & SAG1-Nsi1-s & SAG1-BgIII-as & H7-BgIII-s & H7-PacI-as \\
\hline SVSPtm26 & CWP1-s & CWP-C4-as & SAG1-Nsi1-s & SAG1-BgIII-as & H7-BgIII-s & H7tm-PacI-as \\
\hline GVSPct26 & CWP1-s & CWP-C4-as & GFP-Nsi1-s & GFP-BgIII-as & H7-BgIII-s & H7-PacI-as \\
\hline
\end{tabular}

SAG1-Nsil-s: GTatgcatCTGAGTAGCCGGGCTATGA; GFP-NsiI-s: GCGatgcatAAAGGAGAAGAAC; GFP-BglII-as: GGAagatctTTTGTATAGTTCATCCATG; H7tm-PacI-as: CGttaattaaTCAGACGAACCACCAGCAGAGGAAG; H7BglII-s: CATagatctAATAGCACCGGCGGCGATAGTG; H7PacI-as: GCttaattaaTCACGCCTTCCCGCGGCAGACGAAC; and H7HA-PacI-as: GCttaattaaTCAGGCGTAGTCTGGGACATCGTATGGGTACGCCTTCCCCGGCAGACGAAC.

Primers used for the generation of sequence elements in the constructs shown in Figure 1 are documented in Table 1.

\section{Brefeldin A and Nocodazole Treatment}

Trophozoites were preencysted for $44 \mathrm{~h}$ and induced to encyst for $10 \mathrm{~h}$. Cells were then exposed to a concentration of $75 \mu \mathrm{M}$ brefeldin A (BFA) (Fluka, Neu-Ulm, Germany) for $70 \mathrm{~min}$ or $22.5 \mu \mathrm{M}$ nocodazole (Fluka) for $3 \mathrm{~h}$, respectively. For further analysis, cells were harvested by chilling culture tubes on ice for $30 \mathrm{~min}$, inverting 10 times, and centrifugation at $800 \times g$.

\section{SDS-PAGE and Immunoblotting}

SDS-PAGE was performed according to Laemmli (1970). Cells were harvested as described above; cell pellets were washed once in

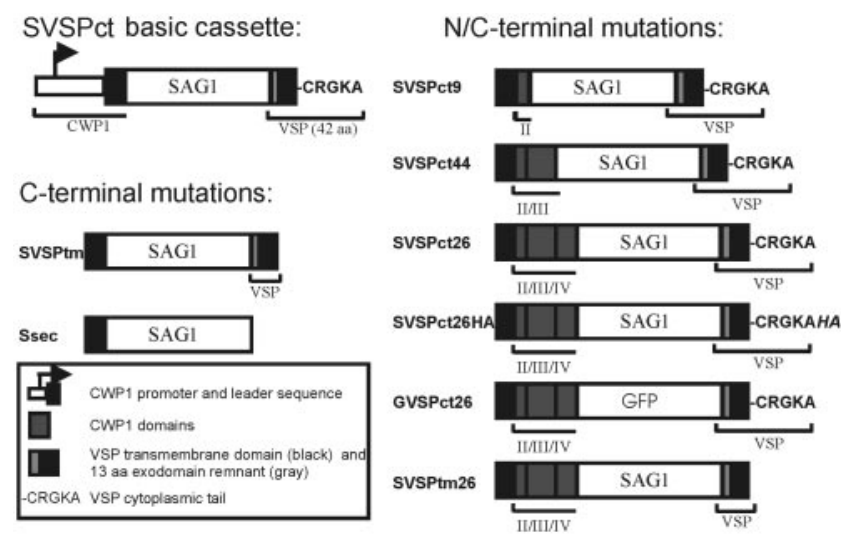

Figure 1. Schematic representation of hybrid genes expressed under the control of the CWP1 promoter. The SVSct basic cassette was used to generate $\mathrm{C}$-terminal variants by mutating or replacing the VSP C-terminus as described in MATERIALS AND METHODS. For the introduction of CWP1 targeting information, insertions of coding sequences were made at the N-terminal side of the predicted reporter protein. A domain color key is provided; CWP1 structural domains are indicated with roman numerals. ice-cold PBS and counted. SDS sample buffer was added to obtain a uniform concentration of $5 \times 10^{5}$ cells/lane, and samples were immediately boiled for $3 \mathrm{~min}$. 10\% polyacrylamide gels were run under reducing conditions with $7.75 \mathrm{mg} / \mathrm{ml}$ dithiothreitol in samples, unless stated otherwise, and proteins were transferred to a nitrocellulose membrane according to standard methods. Antisera were diluted as specified below in PBS, $0.05 \%$ Tween 20 , and $5 \%$ nonfat milk powder. CWP2 was detected with mAb 7D2 (Lujan et al., 1995b) and diluted 1:15,000. SVSPct product was detected by the SAG1-specific mouse mAb DG52 (Bulow and Boothroyd, 1991) and diluted 1:20,000 in nonreducing conditions. Endogenous VSPs were detected with rabbit polyclonal antibody 4A1 (Bruderer et al., 1993), originally raised against a VSP variant expressed by the sheepderived Giardia isolate O2 (Stranden et al., 1990) and diluted 1:10,000. After washing, the nitrocellulose membranes were incubated for $1 \mathrm{~h}$ with a peroxidase-conjugated rabbit anti-mouse or goat anti-rabbit antibody (both Sigma-Aldrich), respectively, and bound antibodies were visualized using the ECL system (NEN, Inc). Densitometric quantification of single bands was performed using ChemiImager 5500 software (Alpha Innotech).

\section{Immunofluorescence Microscopy}

All manipulations were carried out at $4^{\circ} \mathrm{C}$ unless stated otherwise. Trophozoites and encysting cells were harvested as described above, washed twice in ice-cold PBS, and fixed with 3\% paraformaldehyde for $30 \mathrm{~min}$ at room temperature, followed by a 5 -min incubation with $0.1 \mathrm{M}$ glycine in PBS. Fixed cells were permeabilized with $0.1 \%$ Triton X-100 in PBS for 30 min (unless stated otherwise in figure legends) and blocked $>1 \mathrm{~h}$ in $2 \%$ BSA in PBS. Fixed and permeabilized cells were incubated with primary antibodies diluted in $2 \%$ BSA $/ 0.1 \%$ Triton X-100\% in PBS for $1 \mathrm{~h}$. Mouse $\mathrm{mAb}$ DG52 was diluted 1:300 and Texas Red-conjugated mouse mAb A300-TR 1:30 (an anti-CWP antibody; Waterborne, Inc.). GiSar1p, $\mathrm{Gi} \beta^{\prime} \mathrm{COP}$, and GiCLH (clathrin heavy chain) were detected with specific mouse polyclonal antibodies raised against affinity-purified maltose binding protein fusions produced in Escherichia coli (Marti et al., unpublished observations) and diluted 1:300. After washing with ice-cold PBS, cells were incubated for $1 \mathrm{~h}$ with FITC-conjugated sheep antimouse antibody (Sigma-Aldrich). Labeled samples were embedded in Vectashield mounting medium (Vector Laboratories Inc., Burlingame, CA) and viewed with a Leica DM IRBE fluorescence microscope using a 100× HCX PL Fluotar lens (Leica Microsystems Wetzlar GmbH, Wetzlar, Germany). Digital images of 14 optical sections per cell ( $z$-distance, $\sim 0.3 \mu \mathrm{m}$ ) were recorded using a cooled CCD camera (Diagnostic Instruments Inc., Sterling Heights, MI) and processed with the Metaview software package (Visitron Systems GmbH, Puchheim, Germany). 

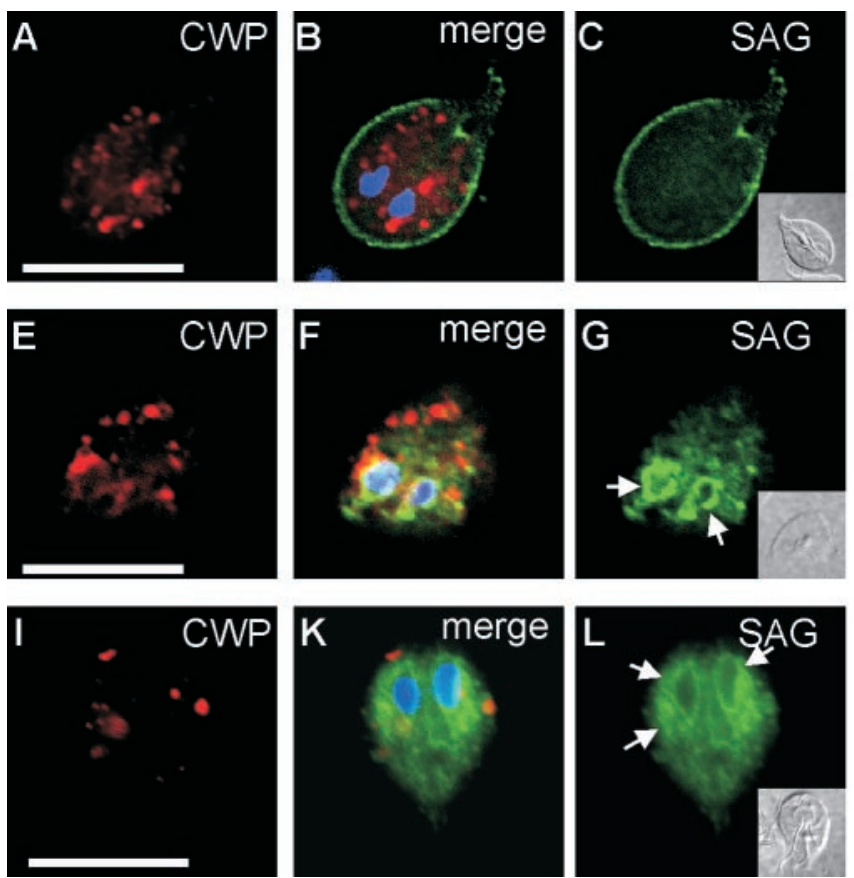
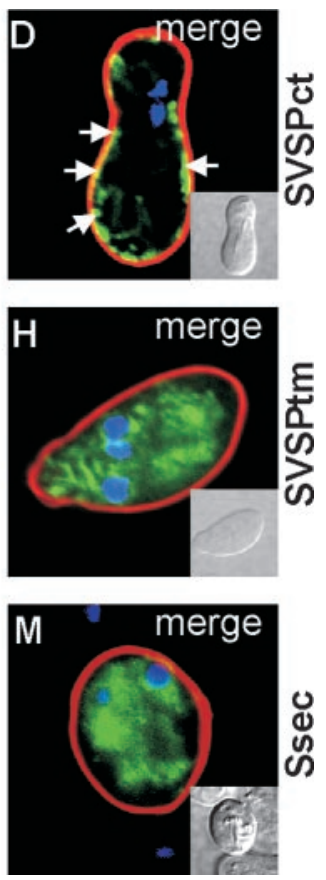

Figure 2. Expression and transport of SVSPct, SVSPtm, and Ssec reporter proteins during Giardia encystation. CWP protein is stained with Texas red (red), SAG1 with FITC (green), and nuclei with DAPI (blue). In encysting trophozoites containing large ESVs (A), SVSPct is localized on the surface membrane (C) and the ER/nuclear envelope compartment. (B) Merged image and DAPI staining. (D) Corresponding colormerged image of a newly developed cyst $<30 \mathrm{~min}$ after secretion of the cyst wall material. SVSPct is still partially associated with the plasma membrane (arrows). (E-G) Removal of the C-terminal pentapeptide from SVSPct (SVSPtm) leads to accumulation of the reporter in internal compartments $(\mathrm{G})$, presumably $\mathrm{ER} /$ nuclear envelope (arrows in G), with no staining of the plasma membrane. (F) Merged images and DAPI staining. ESVs develop normally (E) and cyst formation is unimpaired $(\mathrm{H}$, merged images). (I-M) Further removal of the entire VSP transmembrane domain resulted in accumulation of the now soluble reporter Ssec in the ER/ nuclear envelope compartment $(\mathrm{L}$, arrows). (I) CWP signal alone. (K) Merged images and DAPI staining. (M) Fully developed cyst showing Ssec trapped in endomembrane compartments and nuclear envelope. Insets represent differential interference contrast images of the corresponding cells. Bars, $10 \mu \mathrm{m}$.

\section{Electron Microscopy}

Trophozoites and encysting parasites were grown on $30-\mu \mathrm{m}-$ thick sapphire disks coated with a 5-nm carbon film. The disks, containing a single layer of attached parasites, were plunged into a mixture of liquid propane/ethane $(8 / 2)$ cooled by liquid nitrogen. The ultrarapidly frozen samples were substituted at $-90^{\circ} \mathrm{C}$ in acetone containing $0.5 \%$ osmium tetroxide and $0.25 \%$ glutaraldehyde (Wild et al., 2001) overnight. The temperature was then continuously $\left(5^{\circ} / \mathrm{h}\right)$ raised to $0^{\circ} \mathrm{C}$, and the samples were embedded in epoxy resin at $4^{\circ} \mathrm{C}$. After polymerization at $60^{\circ} \mathrm{C}$ for $2 \mathrm{~d}$, ultrathin sections were cut parallel to the sapphire surface, stained with uranyl acetate and lead citrate, and examined in a CM 12 electron microscope (Philips, Eindhoven, Netherlands) equipped with a slow-scan CCD camera (Gatan, Munich, Germany) at an acceleration voltage of $100 \mathrm{kV}$. Recorded pictures were processed with the Digital Micrograph 3.34 software (Ga$\tan )$.

\section{FACS Analysis}

Transport kinetics of SVSPct product and CWP1 in transgenic cells was measured as follows. Cells were harvested at various times during encystation (see Figure 3 ) and processed for immunofluorescence as described above, without incubation with Triton X-100, before incubation with antibodies. Surface-associated green FITC fluorescence for SVSPct and red TRITC fluorescence for CWP1 were excited using an argon laser at a wavelength of 488 nm (FACSCaliber; Becton Dickinson, Heidelberg, Germany). At least 10,000 cells were analyzed per sample, and each experiment was repeated at least three times. Quantitative data analysis was performed with CELLQuest software (BD Biosciences).

\section{RESULTS}

\section{A Cytoplasmic Pentapeptide Is Required for Export of VSPs}

Efficient and inducible expression of heterologous reporter genes in Giardia under the control of the stage-specific CWP1 promoter has been described previously (Hehl et al., 2000; Marti et al., 2002). Transcription of reporter genes was turned on by inducing encystation of trophozoites in vitro, and expression and reporter trafficking was analyzed in encysting trophozoites during the $\sim 15 \mathrm{~h}$ until secretion of the cyst wall. Only in encysting parasites were both VSP and CWP export pathways active simultaneously, allowing investigation of putative protein-sorting events. By use of this system, regulated expression and correct transport to the plasma membrane of a chimeric protein, SVSPct (Figure 1), have been demonstrated in Giardia (Marti et al., 2002). The SVSPct reporter consisted of a N-terminal leader sequence and a T. gondii SAG1 exodomain attached to a C-terminus derived from the VSP-H7 variant (isolate GS/M-H7) (Nash and Mowatt, 1992), including a membrane-spanning domain and a cytoplasmic tail (CRGKA). This 43-amino-acid $\mathrm{C}$-terminal region is the only sequence that is conserved among all VSPs. Here, we used the SVSPct reporter and two additional C-terminally truncated versions to investigate the relative importance of the VSP-derived domains for reporter export and plasma membrane targeting. The SVSPct protein could be detected by immunofluorescence analysis (IFA) on the surface of transgenic trophozoites and in the ER/nuclear envelope compartment after induction of encystation (Figure 2, A-C). No colocalization of SVSPct and endogenous 
Figure 3. Expression kinetics of SVSPct, CWP, and VSP. (A) Densitometric analysis of SVSPct, CWP, and VSP expression by Western blot during preencystation and the subsequent 24-h encystation period. Maximal levels of the respective proteins were defined as $100 \%$, and data points represent relative total amounts of each antigen (internal and surface combined). SVSPct expression reflects previously observed kinetics and mirrors the similarly induced CWP. Decreased levels of SVSPct and VSP at early points during encystation are caused by complete shedding of surface localized antigen population after induction. (B) FACS analysis of surface exposed SVSPct and CWP during preencystation $(44 \mathrm{~h}, \mathrm{~T}-0)$, induction (at time point 0$)$ with porcine bile and increased pH of culture medium, and encystation for $36 \mathrm{~h}$. Each data point represents the proportion of cells found positive in a FACS analysis (10 ${ }^{4}$ cells) relative to the sample with the maximal percentage of positive cells $(100 \%)$ in the experiment. Parasites for each data point were grown in three separate tubes and mixed before fixation and antibody labeling. Approximately $70 \%$ of cells labeled positive with mAb DG52 at induction of encystation (time point 0 ), indicating export of SVSPct during preencystation.

CWPs was observed in ESVs (in agreement with previous observations) (McCaffery et al., 1994). Cyst wall secretion and assembly was unaffected by SVSPct in the plasma membrane (Figure 2D). In cysts harvested $<30$ min after secretion of the cyst wall, most of the SVSPct protein was still associated with the plasma membrane (Figure 2D); in more mature cysts, the SVSPct signal relocalized to the interior of the cells (data not shown), suggesting active recycling of the plasma membrane protein. To assess the importance of the VSP-derived cytoplasmic tail for export of SVSPct, the CRGKA motif was removed. The resulting reporter, SVSPtm (Figure 1), was not transported to the plasma membrane but rather accumulated in internal membrane compartments, presumably the ER/nuclear envelope (Figure 2, E-H). Complete removal of the VSP-H7 transmembrane domain created a soluble variant of SVSPct (Figure 1, Ssec), which also localized to the ER/nuclear envelope compartment by IFA (Figure 2, I-M). Both truncated reporters contained hydrophobic leader sequences, and both remained in the endomembrane system after cyst wall secretion (Figure 2, $\mathrm{H}$ and $\mathrm{M}$, respectively). The soluble Ssec was not secreted into the culture supernatant during encystation, as determined by Western blot assay (data not shown). This suggested that (1) the C-terminal CRGKA domain performed an essential function in SVSPct trafficking to the plasma membrane and its export from the ER, and (2) that SVSPct was not exported via a default pathway to the plasma membrane but rather by a specific signal-dependent mechanism.

\section{SVSPct and Endogenous CWPs Are Exported with Different Kinetics}

The SVSPct cassette enabled us to induce expression of a reporter with VSP-derived membrane anchor and targeting signals synchronously with endogenous CWPs and thus allowed investigation of protein-sorting events during a period in which both the VSP and CWP export pathways are active. Western blot analysis of total SVSPct protein showed that upregulation of expression followed the kinetics of endogenous CWP expression (Figure 3A) during encystation, with a maximal rate of synthesis between 1 and $14 \mathrm{~h}$ of induction. Surprisingly, a significant amount of total SVSPct protein ( $40 \%$ of maximal amounts) was detectable after $44 \mathrm{~h}$ in preencystation conditions, indicating a leakiness of the CWP promoter in these conditions (data not shown). Immediately after induction of encystation, SVSPct levels dropped significantly before recovering. This is because of a complete shedding and replacement of plasma membrane proteins after a change of medium conditions and is mirrored by the endogenous VSP (Figure 3A). Shedding of SVSPct and recovery could be demonstrated until the late stages of encystation, but export ceased before cyst wall secretion (data not shown). FACS analysis of dually stained cells showed that recovery of SVSPct on the surface is very fast (Figure 3B), most likely drawing on protein synthesized during preencystation. Maximal levels of surface exposed SVSPct were attained in $\leq 1$ hour, whereas secretion of CWP via the regulated ESV pathway began much later in encystation, between 10 and $24 \mathrm{~h}$. Taken together, the completely different export kinetics of synchronously expressed SVSPct and CWP and the lack of common compartments other than the ER provided direct evidence that the respective transport pathways were distinct and did not intersect.

This was also supported by temperature block experiments, which served to further synchronize transport of SVSPct and CWP at the exit from the ER. Cells were encysted at $15^{\circ} \mathrm{C}$ for $14 \mathrm{~h}$ to accumulate proteins in the ER and putative ER exit sites (Figure 4, A-D), and the block was then released at $37^{\circ} \mathrm{C}$ to allow pulsed export of cargo proteins (Figure 4, E-H). In other eukaryotes, coat protein (COP) II vesicle formation at the ER exit still occurs at $15^{\circ} \mathrm{C}$, but further transport is inhibited (Saraste et al., 1986; Milgram and Mains, 1994). After encystation at $15^{\circ} \mathrm{C}$, IFA showed the almost completely overlapping localization of accumulated SVSPct and CWP in the clamp-shaped ER/ 

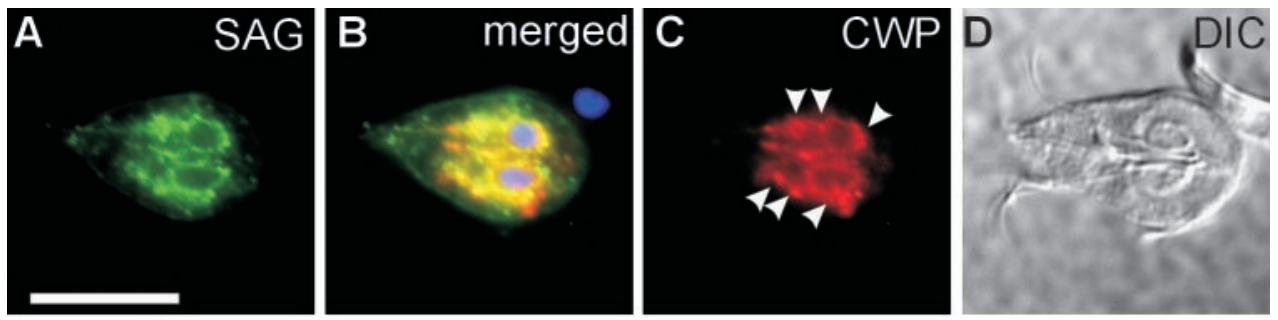

Encysting $15^{\circ} \mathrm{C}$
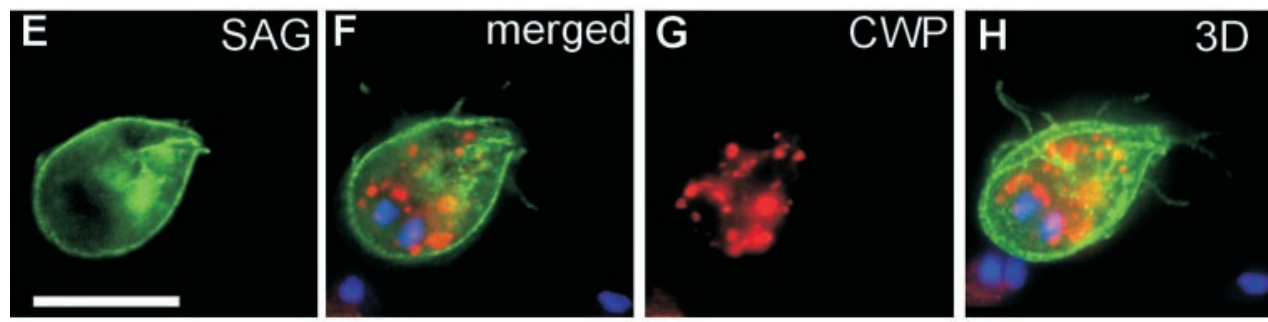

Encysting $15^{\circ} \mathrm{C}->37^{\circ} \mathrm{C}$
Figure 4. Pulsed ER export of CWPs and SVSPct reporter after accumulation in the $\mathrm{ER} /$ nuclear envelope compartment at $15^{\circ} \mathrm{C}$. (A-C) ER/nuclear envelope accumulation of SVSPct. CWP colocalizes with SVSPct at the ER/nuclear envelope and also with putative tER elements as represented by punctate areas along the ER (arrowheads). (B) Merged images and DAPI staining of nuclei; (D) differential interference contrast image of the cell. A release of the temperature block $\left(15\right.$ to $37^{\circ} \mathrm{C}$, $\mathrm{E}-\mathrm{G})$ causes export of SVSPct $(\mathrm{G})$ to the plasma membrane and CWP (E) to ESVs within minutes. No common post-ER compartments are evident. (F) Merged SVSPct, CWP, and DAPI images; $(\mathrm{H}) 3 \mathrm{D}$ reconstruction of all 14 merged optical sections. Bars, $10 \mu \mathrm{m}$. nuclear envelope compartment, in addition to putative transitional ER (tER) elements represented by punctate areas along the ER (Figure 4, A-D). Note that SVSPct is absent from the plasma membrane as a result of stripping of the membrane when the encystation medium was added. Release of the transport block by warming the cells to $37^{\circ} \mathrm{C}$ resulted in formation of ESVs and appearance of SVSPct at the plasma membrane within $20 \mathrm{~min}$ (Figure 4, E-H). No common post-ER compartments could be detected in these cells. These results suggested that SVSPct traveled along a direct route to the plasma membrane. Four separate indications supported the notion that SVSPct export accurately reflected that of endogenous VSPs: (1) both proteins were targeted exclusively to the plasma membrane; (2) correct SVSPct export was dependent on the conserved VSP Cterminus; (3) both were excluded from ESVs and other post-ER endomembrane compartments that could be resolved by IFA; and (4) in pulsed-release or surface-stripping experiments, VSPs (e.g., Figure 3A) and SVSPct (e.g., Figure 4) were replenished with fast kinetics at the surface.

The temperature block experiment also demonstrated for the first time that ESV formation depended directly on ER export of CWPs and, consequently, that sorting of proteins to either of the two pathways occurred during or after ER exit but before CWPs accumulated in ESVs.

\section{Generation of Transgenic Cells with an Ancillary Sorting Phenotype}

Synchronization of ER export indicated that sorting of a reporter with VSP targeting signals (SVSPct) and endogenous CWPs during trafficking along their respective pathways occurred in the early secretory pathway. However, the molecular mechanisms of these events were still unclear. How do targeting signals on CWP and SVSPct confer the necessary selectivity for entering specific transport pathways? To address this question, we generated chimeric con- structs on the basis of the SVSPct gene, which contained competing targeting signals for both export pathways. We hypothesized that this "tug-of-war" situation might lead to either deceleration of SVSPct export to the plasma membrane or sorting of the reporter to the ESV pathway if the CWP-derived targeting signals became dominant. Sequences coding for CWP1 domain II (necessary for sorting into ESVs), the combined domains II and III (necessary for incorporation into the cyst wall), or the entire CWP1 ORF (domains II-IV) were fused in front of the SAG1 reporter gene in pSVSPct to generate constructs SVSPct9, VSPct44, and SVSPct26 (Figure 1). Only the SVSPct26 protein was produced correctly, however; expression of SVSPct9 and VSPct44 resulted in misfolded products, which were not exported (data not shown). The SVSPct26 product was expressed after induction of encystation and accumulated in membrane compartments in the cytoplasm by IFA (Figure 5, A-D) but was undetectable in the plasma membrane. The SVSPct26 protein in these vesicles was secreted quantitatively, like endogenous CWP, and was incorporated into the cyst wall (Figure 5, F-I). Because SVSPct26 appeared to be exported with kinetics identical to those of CWPs and in vesicles indistinguishable from ESVs, we concluded that the reporter was in fact routed through the regulated export pathway via ESV compartments. The CWP targeting signal in SVSPct26 was therefore dominant, redirecting the reporter to the cyst wall. More surprisingly, in regions of transgenic cells in which individual vesicles could be sufficiently resolved in the $z$-plane, the SVSPct26 reporter was found in separate ESVs that did not contain the endogenous CWP detected with our mAb (Figure 5, E, E1, and E2). It is also worth noting that all cells expressing SVSPct26 exhibited this phenotype and that the number of ESVs was approximately twice that observed in wild-type cells (i.e., $\sim 35-50$ ESVs instead of $\sim 20-25$ per cell, respectively). These observations suggested that export of SVSPct26 from the ER 


\section{SVSPct26}
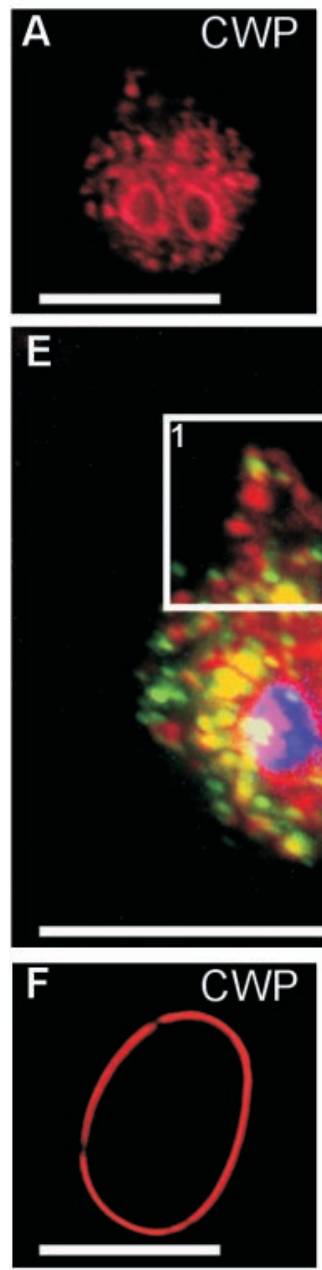

Figure 5. SVSPct26, but not SVSPct26HA, is sorted into distinct ESVs devoid of CWP. (A-D) IF micrograph of a representative encysting cell (layer 7 of 14) using mAbs against SAG1 and CWP. (A) CWP localized in numerous ESVs; SVSPct26 is detected in separate compartments $(C)$ except at possible ER exit sites; merged image and DAPI staining of nuclei (B); differential interference contrast image of cell (D). (E) A threedimensional reconstruction was made of all 14 color-merged optical sections through the cell $(z-$ distance between layers, $0.4 \mu \mathrm{m}$ ). Enlarged areas in more peripheral regions where ESVs can be resolved sufficiently show distinct segregation of the two proteins in separate compartments (E1 and E2). Presentation of a typical mature cyst in a SVSPct26 expressing population (F-I). Distribution of CWP (F) and SVSPct26 (H) shows distinct areas in which only one protein is present in the cyst wall (section 8 of 14). ESV contents are completely secreted, demonstrating that SVSPct26 is exported in distinct but functionally equivalent ESV. (G) merged CWP and SVSPct26 images and DAPI staining of nuclei. (I) Three-dimensional reconstruction of all 14 layers showing discrete areas of SVSPct26 deposition in the cyst wall. ( $\mathrm{J}$ and $\mathrm{K}$ ) Typical encysting cell expressing SVSPct26HA, which is exported together with the CWP detected with the mAb in the same ESVs to the extracellular matrix of mature cysts (NQ). Bars, $10 \mu \mathrm{m}$.
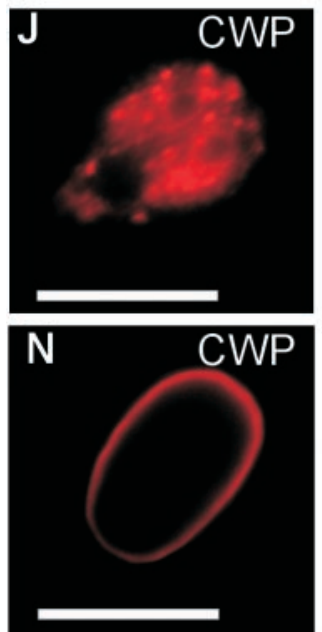
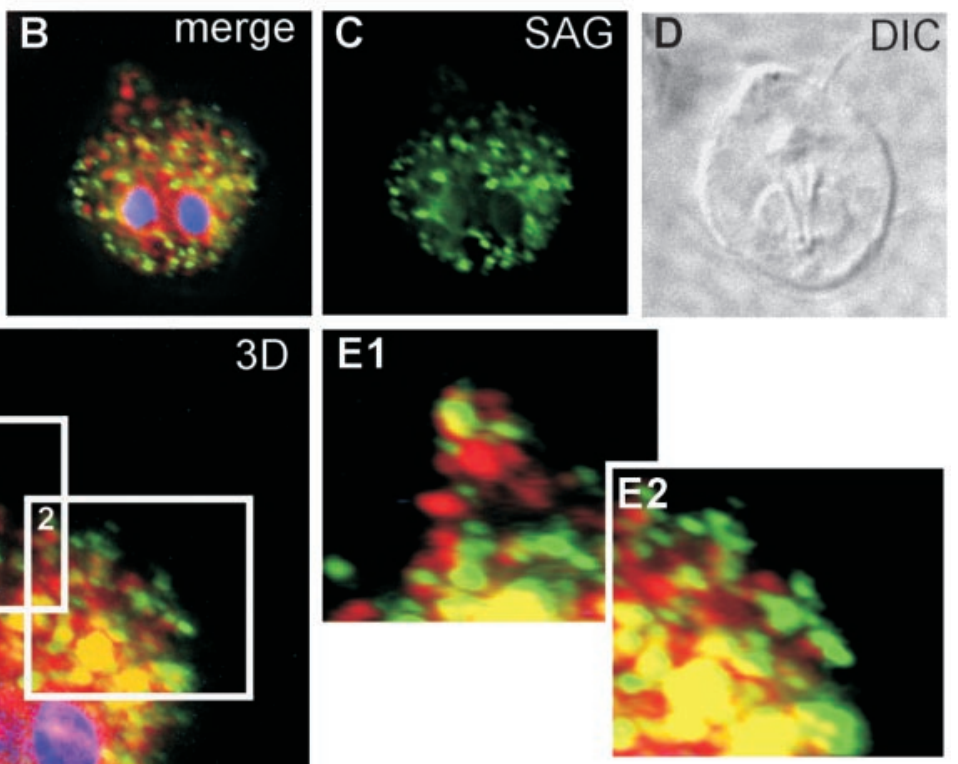

\section{SVSPct26HA}
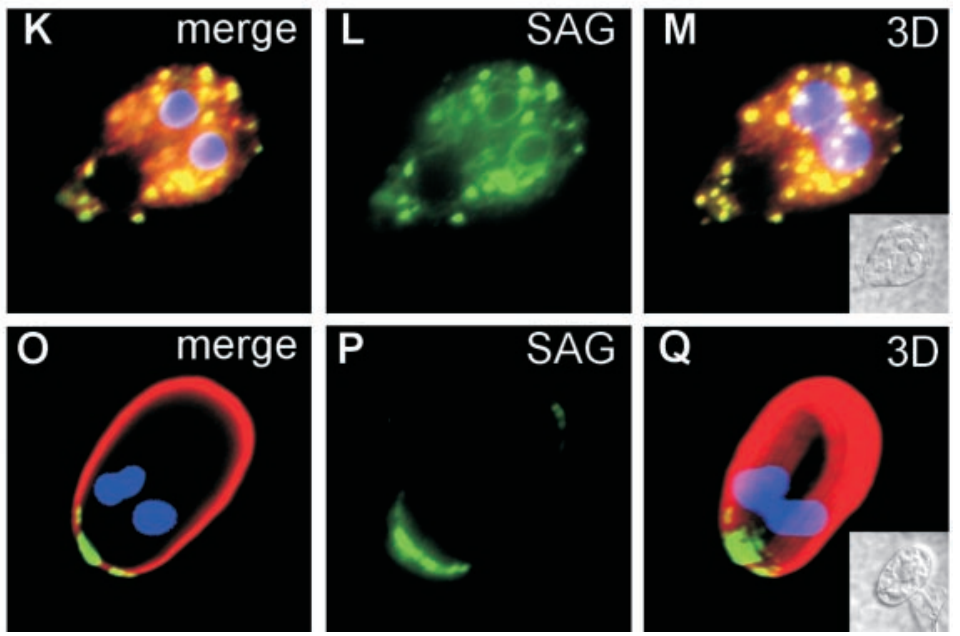
led to generation of an additional class of ESVs, which were devoid of the endogenous CWP detected with our mAb. Was this sorting event artificial, i.e., mediated by the membrane anchoring of the reporter alone, or was this indeed dependent on a functional VSP-derived targeting sequence? To address this question, we engineered a reporter lacking the cytoplasmic tail (SVSPtm26). Expression of SVSPtm26 was lethal, however. As an alternative approach, we tested whether "masking" of the short cytoplasmic CRGKA sequence with an HA tag (SVSPct26HA) would reverse this sorting phenotype. Indeed, in all transgenic cells expressing CWP and SVSPct26HA, the proteins were cotransported (Figure 5, J-M) in a single class of ESVs. This provided strong evidence that sorting of SVSPct26 into distinct ESVs was dependent on the presence of a cytoplasmic VSP targeting signal. In a separate experiment, we obtained direct evidence that the VSP targeting signal was still fully functional in the context of the SVSPct26 protein. Although SVSPct26 was strictly confined to the ESV pathway and the cyst wall after induction of encystation (Figures 5 and 6, $\mathrm{G}-\mathrm{H})$, the reporter was targeted to the plasma membrane in preencysting cells (Figure 6, A-F). As discussed previously, substantial levels of endogenous CWPs and reporter proteins under the control of a CWP1 promoter were observed during preencystation. For example, SVSPct was efficiently exported to the plasma membrane (see also Figure 3), whereas endogenous CWPs accumulated in the ER, most likely because of the absence of a cargo receptor during this stage of differentiation. This also strongly suggested that in cells that had not received a bona fide signal to encyst, CWPs were unable to exit the ER. Taken together, the data show that the targeting signals on SVSPct26 are fully functional and representative of their respective endogenous proteins, CWP or VSP, in terms of their route of transport and their final destination. Moreover, the reporter can be rerouted to either pathway according to the ability of the transport machinery to interpret these targeting signal during development.

After their secretion, both the SVSPct26 and SVSPct26HA proteins aggregated and accumulated in distinct patches in the cyst wall after secretion (Figure 5, F-I, N-Q). Apparently, the final distribution of the reporters in the extracellular matrix was independent of their mode of transport but rather a function of the heterologous SAG1 domain. Although irrelevant for our analysis, the significance of the SAG1 exodomain for aggregation was verified by replacing this domain in SVSPct26 with GFP (GVSPct26, Figure 1). This reporter was secreted in distinct ESVs but distributed homogeneously in the cyst wall after secretion (data not shown).

The subcellular localizations of all reporters used in this study are summarized in Table 2 .

\section{Are CWPs and SVSPct26 Sorted at the ER Exit?}

The previous results in temperature-blocked encysting cells expressing SVSPct showed that differentially labeled punctate structures were present along the ER, indicating that sorting of SVSPct cargo to the VSP pathway may occur during or shortly after export from the ER. We investigated the CRGKA-dependent sorting event further in SVSPct26expressing cells. Here, we had the unique opportunity to track CWP and SVSPct26 export simultaneously and to de-
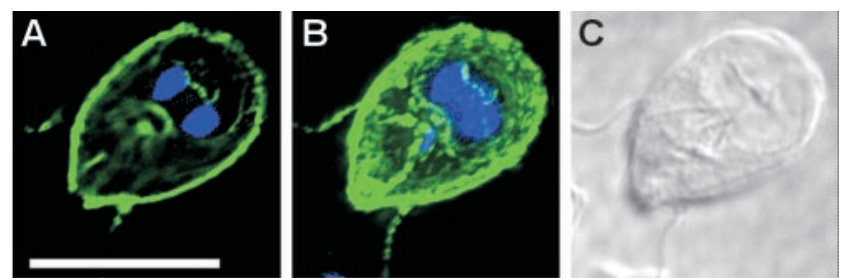

\section{Pre-encysting surface}
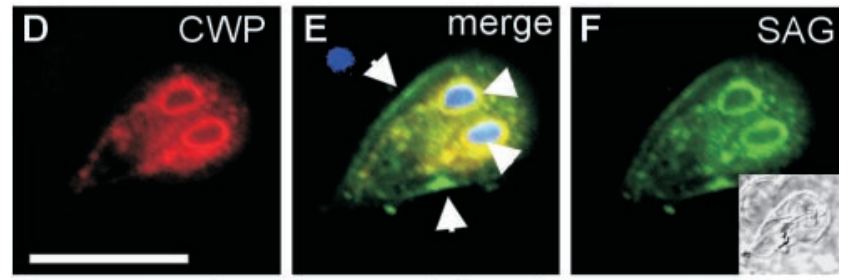

Pre-encysting permeabilized
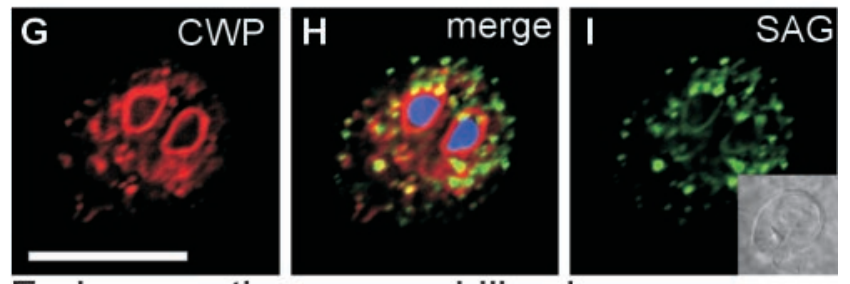

Early encysting permeabilized

Figure 6. SVSPct26 is rerouted during stage differentiation. (A-C) In preencysting cells, SVSPct26 is transported to the outer face of the plasma membrane. (A) Single optical section (6 of 14) and (B) three-dimensional reconstruction of all 14 layers of a representative nonpermeabilized cell incubated with anti-SAG1 antibody. Note staining of flagella, which is diagnostic for surface exposition of an antigen. (C) Differential interference contrast image. (D-F) A cell from the same preparation permeabilized with Triton X-100 and incubated with anti-CWP (D) and anti-SAG1 (F) mAbs. (E) Colocalization of CWP and SVSPct26 in ER/nuclear envelope compartments is evident in the merged images. Arrowheads show nuclear envelope staining. Only SVSPct26 was exported to the surface membrane (arrows in E). Induction of encystation leads to complete redirection of SVSPct26 sorting into the ESV pathway. ER/nuclear envelope staining and developing ESVs containing CWP (G) or SVSPct26 (I). (H) Merged images and DAPI staining of nuclei. Insets, DAPI images of cells. Bars, $10 \mu \mathrm{m}$.

termine at which point the VSP targeting signal in SVSPct26 exerted its effect. To accumulate SVSPct 26 and CWP cells in the ER and ER-exit sites, cells were encysted at $15^{\circ} \mathrm{C}$. In IFA experiments, extensive colocalization of both proteins was observed in the nuclear envelope/ER compartment (Figure 7, A-E). Importantly, the putative punctate tER sites were significantly larger and more pronounced than similar structures observed in temperature-blocked pSVSPct-transformed cells with (see Figure 4). In merged images, these node-like structures appeared as a region of dual staining (yellow) flanked by regions of either CWP (red) or SVSPct26 (green) staining alone (Figure 7, E1-E4). The orientation and distribution of these singly stained regions in the images (top to bottom or left to right) were variable and thus not an artifact of image processing. These structures were inter- 
Table 2. Subcellular localizations of reporter proteins used in this study

\begin{tabular}{|c|c|c|c|c|c|c|c|}
\hline \multirow[b]{2}{*}{ Chimera } & \multicolumn{2}{|c|}{ Export pathway } & \multicolumn{2}{|c|}{ Final localization } & \multicolumn{3}{|c|}{ Construct details } \\
\hline & $\begin{array}{l}\text { ESV } \\
\text { route }\end{array}$ & $\begin{array}{l}\text { VSP } \\
\text { route }\end{array}$ & Surface & ER/Endomembranes & $\begin{array}{l}\text { N-terminal } \\
\text { domains }\end{array}$ & Reporter & C-terminal domains \\
\hline SVSPct & & $x$ & PM & & LS & SAG1 & VSP TM+CRGKA \\
\hline SVSPtm & & & & $X$ & LS & SAG1 & VSP TM \\
\hline Ssec & & & & $X$ & LS & SAG1 & none \\
\hline SVSPct26 & $X^{a}$ & $X^{\mathrm{b}}$ & $\mathrm{PM} / \mathrm{CW}$ & & LS+CWPII/III/IV & SAG1 & VSP TM+CRGKA \\
\hline SVSPct26HA & $x$ & n.d. & CW & & LS+CWPII/III/IV & SAG1 & VSP TM+CRGKAHA \\
\hline SVSPct 44 & & & & $x$ & LS+CWPII/III & SAG1 & VSP TM+CRGKA \\
\hline SVSPct9 & & & & X & LS+CWPII & SAG1 & VSP TM+CRGKA \\
\hline SVSPtm26 & & & & lethal & LS+CWPII/III/IV & SAG1 & VSP TM \\
\hline GVSPct26 & $X^{a}$ & n.d. & CW & $X$ & LS+CWPII/III/IV & GFP & VSP TM+CRGKA \\
\hline
\end{tabular}

Localizations of reporters are indicated with $\mathrm{X}$ and/or specified.

ESVs, encystation-specific vesicles; PM, plasma membrane; CW, cyst wall; LS, leader sequence; TM, transmembrane domain; n.d., not done; CWP1 domains are indicated by roman numerals.

a Transport in separate ESVs.

${ }^{\mathrm{b}}$ Export route only during pre-encystation.

preted as accumulated cargo at putative tER sites and/or small transport intermediates close to ER exit sites. Therefore, the uneven distribution of the two labels suggested that packaging of CWPs and SVSPct26 reporter into separate transport intermediates occurred at specific sites already during ER export. The IFA data also provided the first evidence for the existence of a limited number of ER exit sites in encysting Giardia (represented by the node-like struc- tures). Release of the temperature block at $37^{\circ} \mathrm{C}$ again led to the appearance of segregated ESVs within $<20$ min (data not shown) but was more difficult to interpret because numerous small ESV compartments could not easily be distinguished from the still present ER exit sites. Transgenic parasites at very early time points during encystation, however, showed differentially stained small ESVs (see also Figure 6, G-I), providing additional support for a very early
Figure 7. Accumulation and segregation of secretory cargo at discrete ER exit sites in temperature-blocked SVSPct26-expressing cells. (A and C) Typical appearance of a trophozoite encysted at $15^{\circ} \mathrm{C}$ and probed with anti-CWP and anti-SAG mAbs (section 7 of 14). (B) Three-dimensional reconstruction of 14 merged optical sections through the cell and DAPI staining of nuclei. (D) Differential interference contrast image. (E) Merged images from $\mathrm{A}$ and $\mathrm{C}$ showing node-like accumulation of cargo proteins (numbered squares and arrowheads) at the nuclear envelope and along the ER. (E1-E4) Enlarged areas suggesting cargo segregation at these putative ER exit sites. Bars, $10 \mu \mathrm{m}$.
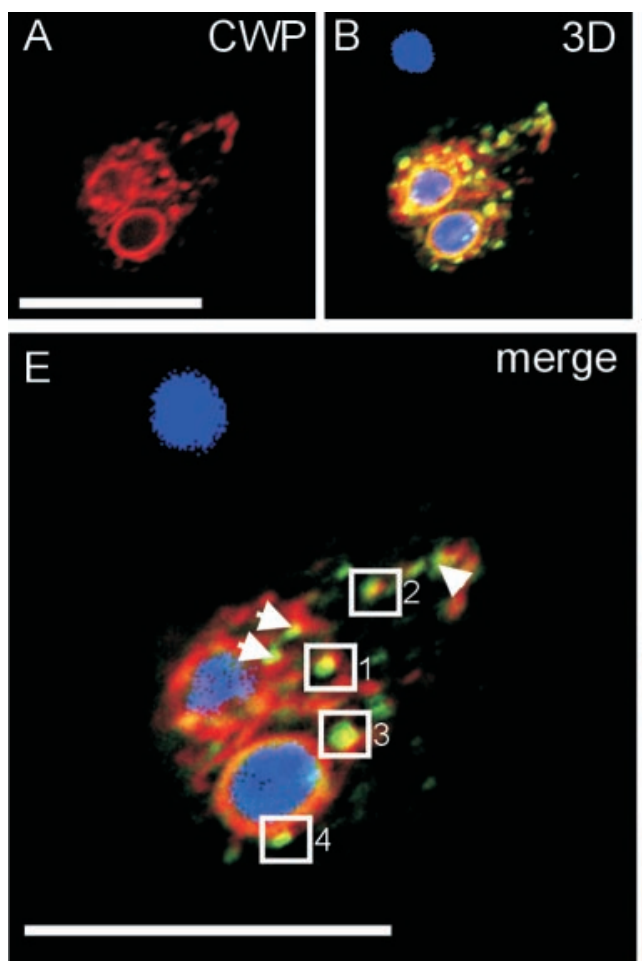
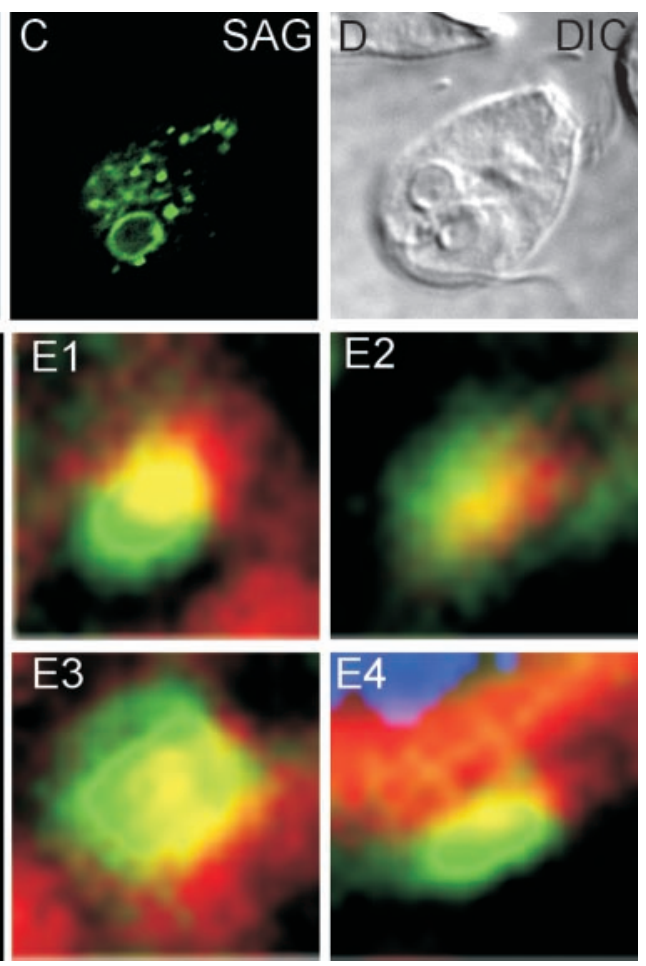

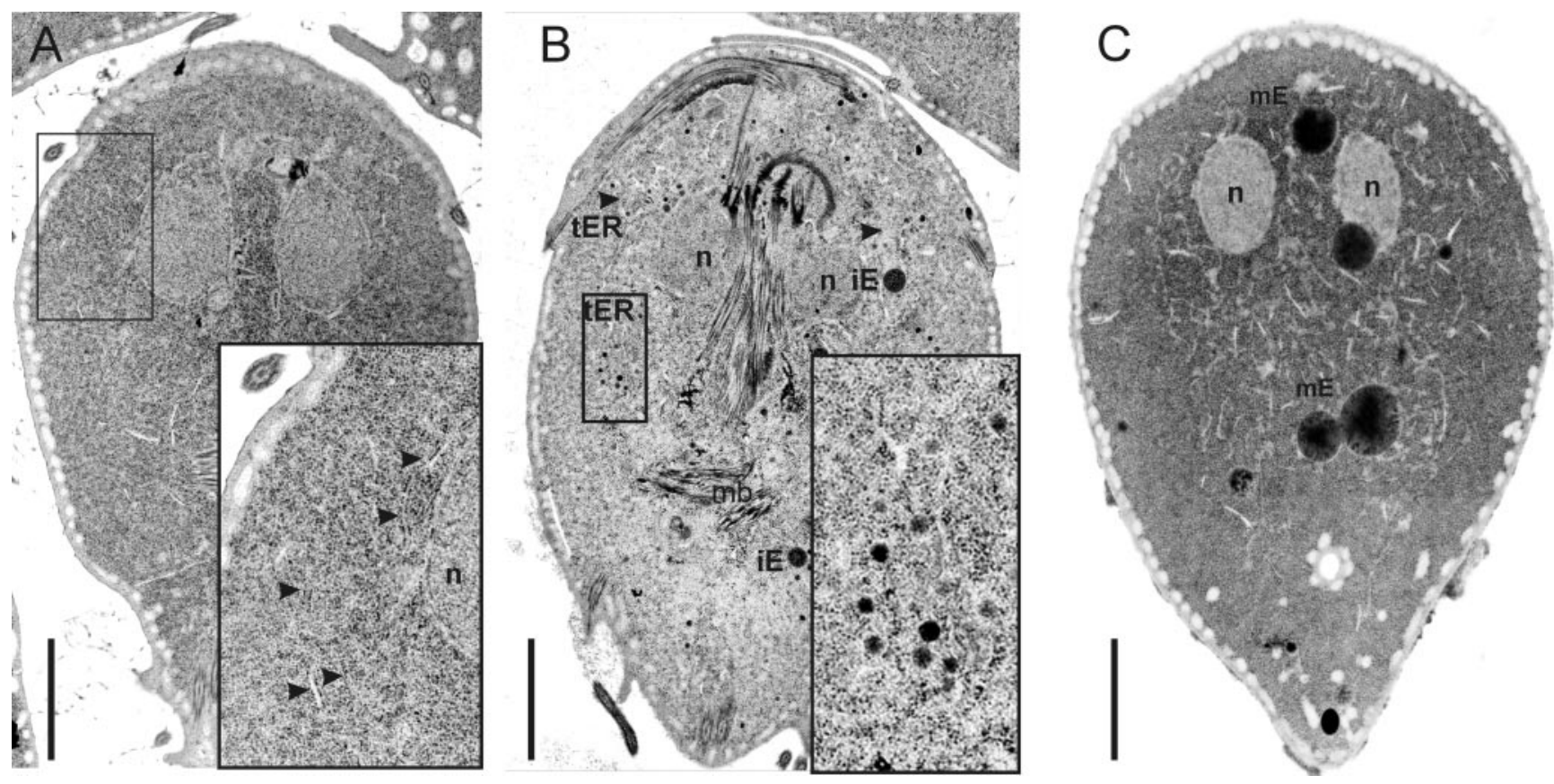

Figure 8. Electron microscopic analysis of ESV development during encystation. Adherent cells were prepared for electron microscopy and sectioned parallel to the ventral plane. (A) Normal trophozoite with distinct nuclear envelope/ER compartments extending bilaterally from the nuclei down to the posterior end of the cell body. No distinct vesicular compartments resembling a Golgi apparatus were detectable. Enlarged area showing mostly ER (A, inset, arrowheads) and the distinct double membrane encasing the nucleus. (B) Early encysting cell (5 $\mathrm{h}$ after induction of encystation) showing the dramatic change in endomembrane compartment morphology in response to the encystation signal. The ER cisternae appear dilated, and at distinct sites, small $(\sim 70 \mathrm{~nm})$ vesicles have developed (B, enlarged area and arrowheads). The putative small transport intermediates contain material of different electron density. At or near these regions, larger ESVs develop, designated immature ESVs (iE). (C) In late encystation (14 h after induction of encystation), small transport vesicles have disappeared and are replaced by large electron-dense ESVs (mature ESVs, $\mathrm{mE}$ ). $\mathrm{n}$, nucleus; mb, median body cytoskeleton structure; iE, immature small ESVs; $\mathrm{mE}$, mature ESVs. Bars, $2 \mu \mathrm{m}$.

SVSPct26-specific sorting event before formation of larger ESVs.

\section{Vesicle Clusters Identified as Putative ER Exit Sites Are Induced during Early Encystation}

Discrete tER elements bilaterally along the ER network had not been identified previously in Giardia. We sought confirmation of this novel observation by performing a comparative transmission electron microscopic survey in trophozoites and early ( $5 \mathrm{~h}$ after induction) and late $(14 \mathrm{~h})$ encysting cells. To obtain consistent aspects of every cell in the preparation and to facilitate identification of structural features, we chose a novel method whereby adherent cells were sectioned in the median plane parallel to the ventral disk. In trophozoites, the extensive bilateral ER network, the nuclear envelope, and PVs were the most prominent endomembrane compartments (Figure 8A). Very few small transport intermediates in the 70-nm range were detected. Conversely, in early encysting cells, distinct clusters of small, variably electron-dense vesicular compartments in close proximity to ER cisterns (Figure $8 \mathrm{~B}$ and insets) were observed. These putative transport intermediates were spherical and of a diameter similar to that of typical COPII vesicles (i.e., $\sim 70 \mathrm{~nm}$ ). The presence and localization of vesicle clusters closely associated with the ER network and nascent ESVs was in agreement with our previous IFA observations and provided strong support for the presence of a limited number of tER sites during early encystation. These novel observations suggested that larger ESVs with a diameter of up to $\sim 1 \mu \mathrm{m}$ (Figure 8C) were formed by successive fusions of these small transport intermediates. This would also explain the paucity of small carrier vesicles (Figure 8C and inset) at these late stages of the encystation process.

\section{ESVs Are Transient Golgi-like Compartments in Giardia}

The existence of discrete induced ER exit sites provided new support for a previously formulated hypothesis that ESVs, generated specifically in response to ER export of cyst wall material, corresponded to Golgi-like compartments (Lujan et al., 1995a; Hehl et al., 2000). We used specific antibodies generated against Giardia COPI or COPII components to test whether these coats were associated with early ESVs and/or transport intermediates near ER exit sites. IFA analysis of encysting cells revealed that GiSar1p, the Giardia orthologue of the COPII-specific GTPase Sar1p (Marti et al., unpublished observations), localized to the clamp-shaped ER/nuclear envelope structure (Figure 9, A-C). Significantly, major concentrations of GiSar1p were observed in distinct posterior areas in early encysting cells, colocalizing with CWP in 

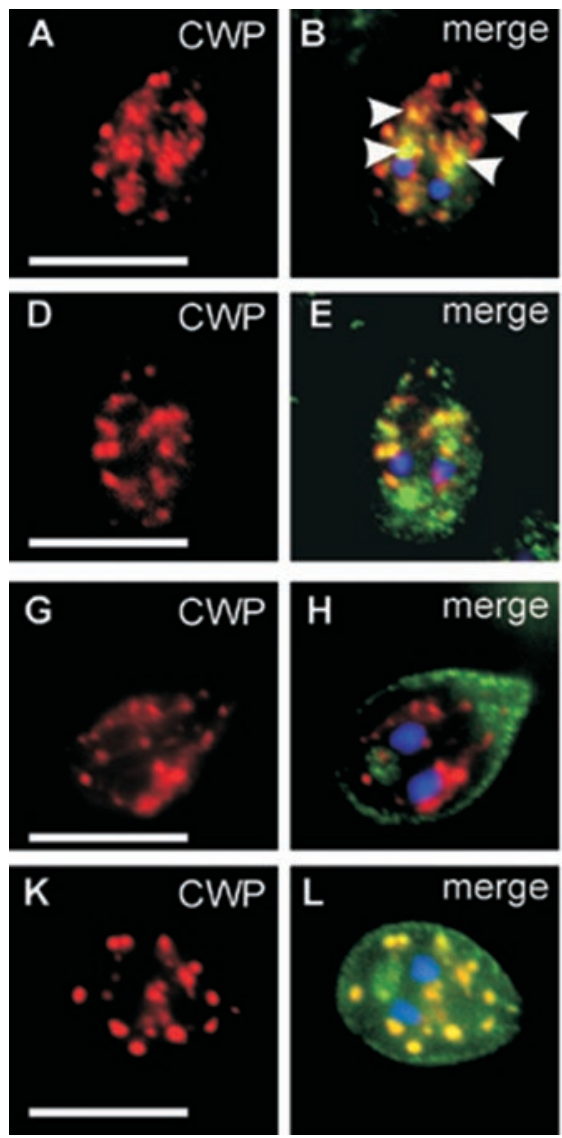
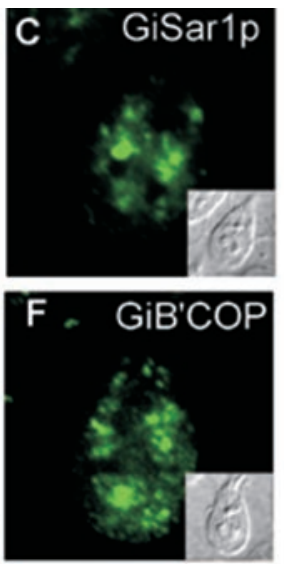
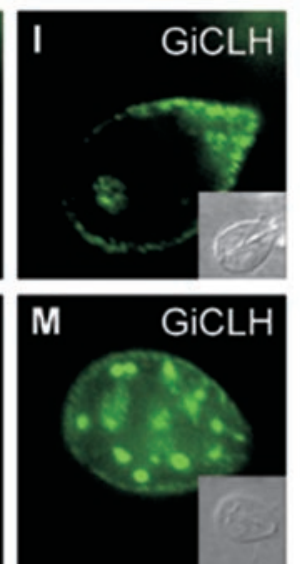
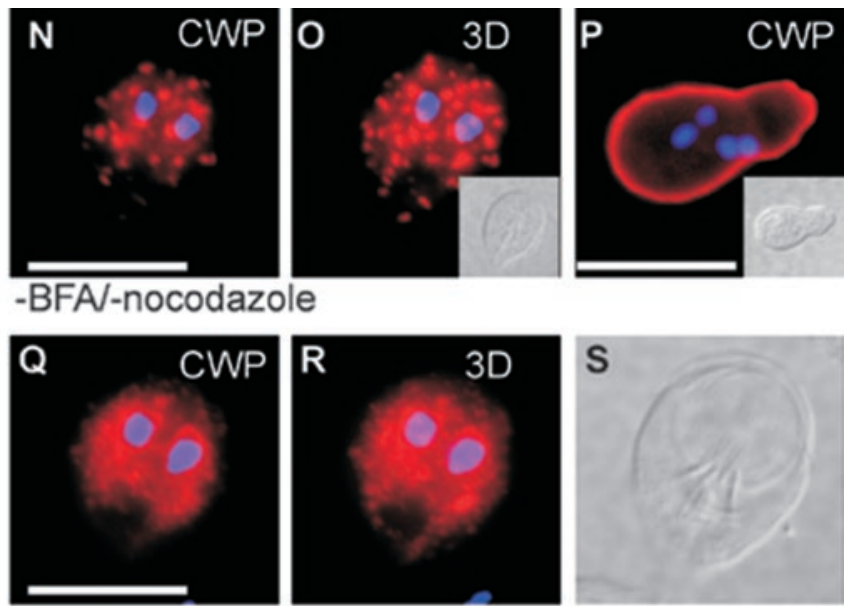

+BFAV-nocodazole
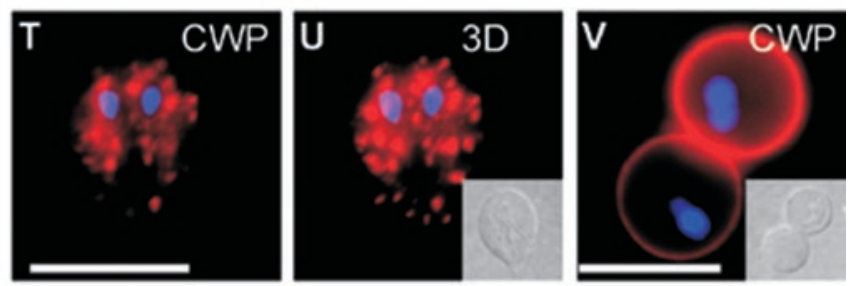

-BFA +nocodazole

Figure 9. ESVs show Golgi characteristics. (A-C) Subcellular localization of GiSar1p, the Giardia orthologue of the COPII-specific GTPase Sar1. A representative encysting cell with numerous ESVs containing cyst wall material, labeled with anti-CWP antibody, is shown. GiSar1p distribution was confined to tER elements along the ER/nuclear envelope compartment. (B) Merged CWP and GiSar1p images show overlapping distribution in distinct posterior areas of the cell where tER elements have been identified (arrowheads). (D-F) Subcellular localization of Gi $\beta^{\prime} \mathrm{COP}$, the Giardia orthologue of the COPI subunit $\beta^{\prime} \mathrm{COP}$ in a representative encysting cell. (D) Encysting cell with ESVs containing cyst wall material, labeled with anti-CWP mAb. (F) Gi $\beta^{\prime} \mathrm{COP}$ localizes to endomembrane structures. (E) Merged Gi $\beta^{\prime} \mathrm{COP}$ and CWP images show that distribution of Gi $\beta^{\prime} \mathrm{COP}$ partially overlaps with the CWP signal in ESVs. Antibodies against GiCLH, the Giardia orthologue of the clathrin heavy chain, label PVs but not ESVs in early encysting cells (G-I, 5 h after induction). (G, I) Subcellular localization of CWP and GiCLH, respectively. (H) Merged GiCLH and CWP images showing no colocalization of the two proteins at this stage of encystation. In late encystation, however, large ESVs are heavily labeled with anti-GiCLH antibodies (K-M). (K and M) Subcellular localization of CWP and GiCLH, respectively. (L) Merged GiCLH and CWP images. (N-P) IFA micrographs of a typical encysting wild-type cell with numerous large ESVs containing cyst wall material, labeled with anti-CWP antibody (N, section 8 of 14). (O) Three-dimensional reconstruction of 14 sections. (P) Typical cyst containing four nuclei (DAPI stain, blue). Cyst formation was completely inhibited in BFA-treated cells (Q-S). Representative encysting trophozoite treated with BFA for $1 \mathrm{~h}$ (Q, section 7 of 14$)$ showing fragmented ESVs and cyst wall material redistributed into the nuclear envelope and bilaterally along the ER. (R) Three-dimensional reconstruction of 14 sections. (S) Differential interference contrast image. (T) Representative encysting wild-type cell treated with nocodazole. ESV morphology and development is normal. (U) Three-dimensional reconstruction of 14 optical sections. (V) Secretion of cyst wall material is normal in nocodazoletreated cells, but cysts show aberrant spherical morphology and nuclear division has not occurred. Insets: Differential interference contrast images of cells. Bars, $10 \mu \mathrm{m}$.

regions in which tER elements had been identified (Figure 9B, arrowheads). Similarly, antibodies against Gi $\beta^{\prime} \mathrm{COP}$, the Giardia orthologue of the COPI subunit $\beta^{\prime} \mathrm{COP}$ (Marti et al., unpublished observations), labeled nascent ESVs and additional small but clearly defined CWP-negative endomembrane structures (Figure 9, D-F). Association of COPI or COPII components with ESVs or ER exit sites, respectively, further confirmed the Golgi characteristics of ESVs, at least during early stages of encystation. If nascent or maturing
ESVs constituted Golgi-like compartments, will they become trans-Golgi-like compartments late in encystation? We addressed this question by investigating association of the Giardia clathrin heavy chain (GiCLH, Marti et al., unpublished observations) with ESV membranes during encystation. Using a specific antibody against GiCLH, we performed IFA in early or late encysting trophozoites. At 3-5 h after induction, GiCLH was localized to PVs and was clearly not associated with compartments containing CWP (Figure 


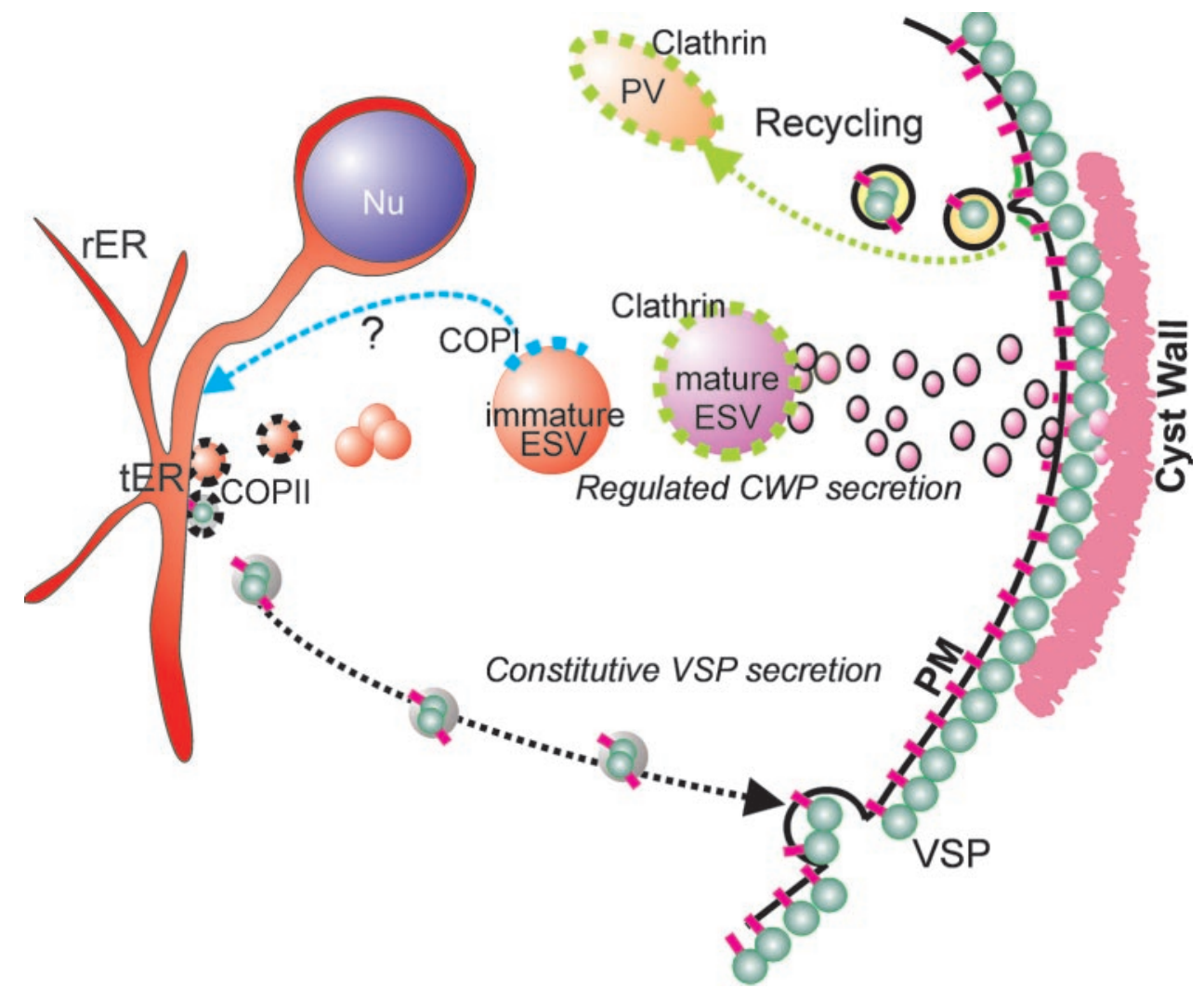

Figure 10. Summary and interpretation of the combined data. The model shows development and maturation of ESVs starting with small tER-derived putative COPII vesicles, which merge to form larger immature ESVs. Separation of CWP-containing vesicles from those containing cargo secreted to the plasma membrane is achieved by selective fusion competency of transport intermediates. Association of small ESVs with COPI suggests maturation by retrograde transport to the ER. According to the data presented here, immature ESVs may therefore represent transient Golgi-equivalents, which develop to clathrin-associated trans-Golgi-like cisterna. Mature ESVs become responsive to an internal signal initiating nuclear division and ESV fragmentation into small carrier vesicles and/or tubules, which fuse with the plasma membrane. After secretion of the extracellular matrix, plasma membrane proteins, such as VSPs, are internalized and presumably transported to the lysosome-like PVs for recycling.
9, G-I). In contrast, in late encysting cells (10 h after induction), antibodies against GiCLH heavily labeled the large mature ESVs in addition to PV compartments (Figure 9, $\mathrm{K}-\mathrm{M})$. These novel observations therefore provided direct evidence for a maturation process during formation of secretion-competent ESVs.

Association of COPI with developing ESVs also explained their sensitivity to the drug BFA. Addition of BFA to encysting cells caused ESVs to disintegrate completely and the CWP label to become distributed along the ER structure (Figure 9, Q-S). In contrast, treatment of encysting cells with the microtubule depolymerizing drug nocodazole, which normally severely alters Golgi morphology, did not affect ESV development or impair encystation of Giardia parasites (Figure 9, T-V).

\section{DISCUSSION}

Many protozoan parasites generate special post-Golgi compartments for regulated secretion of proteins involved in host cell invasion, intracellular replication (e.g., Apicomplexa) (Joiner and Roos, 2002), or the assembly of an extracellular matrix (e.g., Entamoeba) (Ghosh et al., 1999). Similarly, the early-diverged protozoan Giardia intestinalis generates ESVs containing cyst wall material during the encystation process, in addition to transporting VSPs to the plasma membrane in a constitutive manner. Because Giardia has no recognizable Golgi apparatus in all developmental stages, it is unclear how the parasite can accomplish faithful sorting of differently targeted proteins, in particular during encystation. To provide first answers to the problem of protein sorting in a Golgi-less organism, we investigated sorting processes depending on VSP or CWP targeting signals and tracked stage-specifically expressed and differentially targeted reporter proteins during the $\sim 15$-h encystation period in which both proteins are transported simultaneously. We found evidence that two major secretory pathways in Giardia diverged before the generation of ESVs and identified a limited number of discrete and novel tER-like regions of intense secretory transport activity in early encysting cells giving rise to larger ESV compartments.

In a previous study, we had determined that soluble CWP1 was targeted to ESVs and subsequently to the cyst wall by specific N-terminal and central signals on the protein (Hehl et al., 2000). Here, we demonstrated that transport of constitutively expressed membrane-anchored VSPs to the plasma membrane depended on the presence of a conserved cytoplasmic C-terminus. Although the cytoplasmic tail of VSPs was necessary for ER exit and surface targeting of the SVSPct reporter, it remained unclear whether it was also sufficient. Transplantation of the CRGKA sequence to heterologous transmembrane domains has not yet yielded a correctly exported reporter (data not shown). However, this may be simply because of a lack of suitable non-VSP transmembrane regions compatible with plasma membrane insertion (no other plasma membrane proteins have been identified yet in Giardia). 
Most importantly for our investigation, we were able to demonstrate that reporters with VSP or CWP targeting signals reached the predicted destination and were exported with very different kinetics. Export of the SVSPct reporter, like endogenous VSP proteins (McCaffery et al., 1994), followed a route that bypassed ESVs. This was also true in cells in which ER export of SVSPct and CWP had been synchronized by a $15^{\circ} \mathrm{C}$ temperature block with subsequent release of accumulated proteins at $37^{\circ} \mathrm{C}$. Even under these conditions, transport of the SVSPct reporter occurred too fast to determine where the two pathways diverged, i.e., at which level protein sorting was performed. A novel approach to this problem was found by expression of the membrane-anchored SVSPct26 reporter with competing luminal and cytoplasm exposed targeting signals. The principal effect of the SVSPct26 phenotype was that export of endogenous CWP and the reporter were now synchronized and proceeded along the ESV pathway. Our experiments also clearly demonstrated that targeting signals to both the plasma membrane and the ESV / cyst wall pathways were intact (see below). Competing sorting signals in luminal and cytoplasmic domains have been described previously in polarized MDCK cells directing endolyn to lysosomes preferentially via the apical plasma membrane (Ihrke et al., 2001), but they have also been used in chimeric proteins (Jacob et al., 1999). The major difference in Giardia was that protein sorting to the two possible export routes occurred in the absence of a stable Golgi apparatus and not at the trans-Golgi network, as in all other eukaryotes. The observation that SVSPct26 segregated into separate but functionally equivalent ESVs was unexpected and turned out to be the key phenotype of these transgenic parasites. Although CWP-derived targeting signals were dominant after induction of encystation, localization of SVSPct26 at the plasma membrane of preencysting cells provided direct evidence that SVSPct26 contained dual targeting signal and in addition that the Giardia transport system had the ability to interpret targeting signals in a stage-specific manner. Targeting signals for the ESV pathway were thus nonfunctional, in agreement with a lack of CWP export from the ER and emphasizing a requirement for cargo receptors or escorters. Similar modes of coexport to secretory organelles have been found for the T. gondii MIC1 and MIC4 proteins and the Plasmodium RAP2 (Baldi et al., 2000; Reiss et al., 2001). Together, the data indicated that CWP and SVSPct26 were sorted to the ESV pathway in a stagespecific manner by virtue of their CWP-derived motifs but segregated into functionally equivalent but distinct ESVs because of the presence of a functional VSP targeting signal on SVSPct26.

Accumulation of SVSPct26 and CWP in the early secretory pathway of temperature-blocked encysting cells led to the initial identification of potential tER or ER exit sites by IFA. Because no ESVs formed under these conditions, exit sites were readily identifiable as node-like structures of dual staining. Interestingly, closer examination of these areas showed that the two proteins occupied distinct domains, arguing for sorting into separate transport intermediates immediately after export from the ER. Because this sorting function was mediated by the VSP cytoplasmic domain, the observation provided the first evidence for a cargo selection machinery at the ER exit that can distinguish between the VSP export signals and those of soluble CWPs. The simplest explanation was that SVSPct26 protein was packaged into pre-ESV transport vesicles, which contained extra membrane determinants (recruited by the VSP part of the chimera) that allowed only homotypic fusion with identical vesicles but not with those containing genuine CWPs exported from the ER. The molecular basis for this sorting event and whether it indeed represented the sorting of VSPs and CWPs during export, as indicated by the fact that no VSPs or similarly targeted reporters can be detected in ESVs at any time, remain to be determined.

Discrete ER exit sites had never been described in the Giardia literature. We obtained independent confirmation of this idea on a structural level by identifying novel regions containing numerous 70-nm transport intermediates intimately associated with the ER. Significantly, the locations of these "active" areas in transmission electron microscopy matched those of the node-like structures identified in the IFA experiments (compare Figures 7E and $8 \mathrm{~B}$ ). The presence of larger ESVs in these areas also suggested that ESVs arose by fusion of putative small transport intermediates. In animal cells and also in yeast, the interface between ER and Golgi consists of an intermediate compartment, called the ER-Golgi intermediate compartment (Schweizer et al., 1990), which arises by fusion of ER-derived vesicles or, alternatively, is viewed as an independent stable compartment. In lower eukaryotes, there is less support for such an intermediate compartment, but ER-derived vesicles are thought to fuse directly with the cis-most cisterna of the Golgi or to give rise to this cisterna by homotypic fusion (depending on the underlying Golgi model).

What is the nature of ESV compartments if indeed they are generated by fusion of small transport intermediates from these ER exit sites? We showed colocalization of GiSar1p and CWP at tER elements and small ESVs, suggesting that ER-derived vesicles are COPII coated. Many ESVs were associated with Gi $\beta^{\prime} \mathrm{COP}$ by IFA, which argues for an involvement of COPI in ESV-ER retrograde transport and provides an explanation for the BFA sensitivity of ESVs (Lujan et al., 1995a; Hehl et al., 2000). The most striking developmental change in ESV characteristics was the increasing association of Giardia clathrin, GiCLH, with ESV membranes. Recruitment of clathrin to late ESVs could serve to disperse large ESVs into smaller transport vesicles in response to the secretion signal. The data are compatible with the notion of ESVs as maturing Golgi-like cisterna with changing molecular characteristics, albeit without a recognizable sorting function. The transient nature and changing properties of these Golgi-like compartments can be explained on the basis of the cisternal maturation model (Allan and Balch, 1999), with the important and unique difference that the putative cis-, median-, and trans-Golgi equivalents in encysting Giardia are separated in time and not in space. The most obvious function of such a transiently present "Golgi equivalent" is to provide a compartment dedicated to the posttranslational modification and maturation of cyst wall material. In Figure 10, we propose a model summarizing the insights into mechanisms of protein sorting to the two 
major secretory pathways in Giardia and provide a rationale for the neogenesis and maturation of ESVs. Exported proteins are sorted according to their targeting signal into distinct COPII vesicles (black coats), possibly already at the exit from the ER. VSPs may directly recruit coat components via their cytoplasmic tail, whereas the soluble CWPs are most likely dependent on cotransport with other (transmembrane) protein(s) providing this function (i.e., cargo receptor/escorter). Only transport intermediates containing CWPs are able to fuse homotypically and give rise to the larger ESV compartments. Early ESVs mature in situ by COPI-dependent retrograde transport (blue coat) and processing/modification of cargo. Clathrin associates with mature ESVs (green coats), which become receptive to a signal triggering secretion of the cyst wall material. This implies that the status of ESVs goes from "loaded" to "cocked" during late encystation and that this transition is determined by factors associated with the ESV membrane. Small VSP-containing cargo vesicles, conversely, appear to travel directly from the ER to the plasma membrane without fusing with another membrane compartment along the way. This is consistent with the low complexity of the Giardia secretory system on a molecular (Marti et al., unpublished observations) and morphological level.

Elucidation of the exact nature of ESVs is directly linked to the question of Golgi neogenesis in Giardia and has broad implications. If ESVs are indeed an ancient Golgi equivalent generated only for transport and possibly modification of cyst wall material, Giardia is the only experimentally accessible organism in which neogenesis of this organelle can be studied directly. Hence, this protozoan is an invaluable model to study the "old rules" of secretory transport in a primordial eukaryotic setting accessible to the modern tools of cell biology.

\section{ACKNOWLEDGMENTS}

The authors thank Dr. Theodore Nash (National Institutes of Health, Bethesda, MD) for the monoclonal cyst wall protein 2 antibody 7D2, Dr. Frank Seeber (Philipps-Universität, Marburg, Germany) for the plasmids SAG1-GPI and SAG1-TM, Dr. J.C. Boothroyd for mAb DG52 against SAG1, and Therese Michel for expert technical assistance. This work was supported by grant 31-58912.99 of the Swiss National Science Foundation. Y.L. was supported by a training grant from the China Scholarship Council.

\section{REFERENCES}

Allan, B.B., and Balch, W.E. (1999). Protein sorting by directed maturation of Golgi compartments. Science 285, 63-66.

Baldi, D.L., Andrews, K.T., Waller, R.F., Roos, D.S., Howard, R.F., Crabb, B.S., and Cowman, A.F. (2000). RAP1 controls rhoptry targeting of RAP2 in the malaria parasite Plasmodium falciparum. EMBO J. 19, 2435-2443.

Boucher, S.E., and Gillin, F.D. (1990). Excystation of in vitro-derived Giardia lamblia cysts. Infect. Immun. 58, 3516-3522.

Bruderer, T., Papanastasiou, P., Castro, R., and Kohler, P. (1993). Variant cysteine-rich surface proteins of Giardia isolates from human and animal sources. Infect. Immun. 61, 2937-2944.
Bulow, R., and Boothroyd, J.C. (1991). Protection of mice from fatal Toxoplasma gondii infection by immunization with p30 antigen in liposomes. J. Immunol. 147, 3496-3500.

Dacks, J.B., and Doolittle, W.F. (2001). Reconstructing/deconstructing the earliest eukaryotes: how comparative genomics can help. Cell 107, 419-425.

Feely, D.E., and Dyer, J.K. (1987). Localization of acid phosphatase activity in Giardia lamblia and Giardia muris trophozoites. J. Protozool. 34, 80-83.

Ghosh, S.K., Field, J., Frisardi, M., Rosenthal, B., Mai, Z., Rogers, R., and Samuelson, J. (1999). Chitinase secretion by encysting Entamoeba invadens and transfected Entamoeba histolytica trophozoites: localization of secretory vesicles, endoplasmic reticulum, and Golgi apparatus. Infect. Immun. 67, 3073-3081.

Hehl, A.B., Marti, M., and Kohler, P. (2000). Stage-specific expression and targeting of cyst wall protein-green fluorescent protein chimeras in Giardia. Mol. Biol. Cell 11, 1789-1800.

Ihrke, G., Bruns, J.R., Luzio, J.P., and Weisz, O.A. (2001). Competing sorting signals guide endolyn along a novel route to lysosomes in MDCK cells. EMBO J. 20, 6256-6264.

Jacob, R., Preuss, U., Panzer, P., Alfalah, M., Quack, S., Roth, M.G., Naim, H., and Naim, H.Y. (1999). Hierarchy of sorting signals in chimeras of intestinal lactase-phlorizin hydrolase and the influenza virus hemagglutinin. J. Biol. Chem. 274, 8061-8067.

Joiner, K.A., and Roos, D.S. (2002). Secretory traffic in the eukaryotic parasite Toxoplasma gondii: less is more. J. Cell Biol. 157, 557-563.

Laemmli, U.K. (1970). Cleavage of structural proteins during the assembly of the head of bacteriophage T4. Nature 227, 680-685.

Lujan, H.D., Marotta, A., Mowatt, M.R., Sciaky, N., LippincottSchwartz, J., and Nash, T.E. (1995a). Developmental induction of Golgi structure and function in the primitive eukaryote Giardia lamblia. J. Biol. Chem. 270, 4612-4618.

Lujan, H.D., Mowatt, M.R., Conrad, J.T., Bowers, B., and Nash, T.E. (1995b). Identification of a novel Giardia lamblia cyst wall protein with leucine-rich repeats: implications for secretory granule formation and protein assembly into the cyst wall. J. Biol. Chem. 270, 29307-29313.

Marti, M., Li, Y., Kohler, P., and Hehl, A.B. (2002). Conformationally correct expression of membrane-anchored Toxoplasma gondii SAG1 in the primitive protozoan Giardia duodenalis. Infect. Immun. 70, 1014-1016.

McCaffery, J.M., Faubert, G.M., and Gillin, F.D. (1994). Giardia lamblia: traffic of a trophozoite variant surface protein and a major cyst wall epitope during growth, encystation, and antigenic switching. Exp Parasitol 79, 236-249.

Milgram, S.L., and Mains, R.E. (1994). Differential effects of temperature blockade on the proteolytic processing of three secretory granule-associated proteins. J. Cell Sci. 107, 737-745.

Mowatt, M.R., Lujan, H.D., Cotten, D.B., Bowers, B., Yee, J., Nash, T.E., and Stibbs, H.H. (1995). Developmentally regulated expression of a Giardia lamblia cyst wall protein gene. Mol. Microbiol. 15, 955-963.

Nash, T.E., and Mowatt, M.R. (1992). Characterization of a Giardia lamblia variant-specific surface protein (VSP) gene from isolate GS/M and estimation of the VSP gene repertoire size. Mol. Biochem. Parasitol. 51, 219-227.

Papanastasiou, P., Hiltpold, A., Bommeli, C., and Kohler, P. (1996). The release of the variant surface protein of Giardia to its soluble isoform is mediated by the selective cleavage of the conserved carboxy-terminal domain. Biochemistry 35, 10143-10148. 
Reiner, D.S., Douglas, H., and Gillin, F.D. (1989). Identification and localization of cyst-specific antigens of Giardia lamblia. Infect. Immun. 57, 963-968.

Reiss, M., Viebig, N., Brecht, S., Fourmaux, M.N., Soete, M., Di Cristina, M., Dubremetz, J.F., and Soldati, D. (2001). Identification and characterization of an escorter for two secretory adhesins in Toxoplasma gondii. J. Cell Biol. 152, 563-578.

Saraste, J., Palade, G.E., and Farquhar, M.G. (1986). Temperaturesensitive steps in the transport of secretory proteins through the Golgi complex in exocrine pancreatic cells. Proc. Natl. Acad. Sci. USA 83, 6425-6429.

Schweizer, A., Fransen, J.A., Matter, K., Kreis, T.E., Ginsel, L., and Hauri, H.P. (1990). Identification of an intermediate compartment involved in protein transport from endoplasmic reticulum to Golgi apparatus. Eur. J. Cell Biol. 53, 185-196.
Sogin, M.L., Gunderson, J.H., Elwood, H.J., Alonso, R.A., and Peattie, D.A. (1989). Phylogenetic meaning of the kingdom concept: an unusual ribosomal RNA from Giardia lamblia. Science 243, 75-77.

Soltys, B.J., Falah, M., and Gupta, R.S. (1996). Identification of endoplasmic reticulum in the primitive eukaryote Giardia lamblia using cryoelectron microscopy and antibody to Bip. J. Cell Sci. 109, 19091917.

Stranden, A.M., Eckert, J., and Kohler, P. (1990). Electrophoretic characterization of Giardia isolated from humans, cattle, sheep, and a dog in Switzerland. J. Parasitol. 76, 660-668.

Sun, C.H., Chou, C.F., and Tai, J.H. (1998). Stable DNA transfection of the primitive protozoan pathogen Giardia lamblia. Mol. Biochem. Parasitol. 92, 123-132.

Wild, P., Schraner, E.M., Adler, H., and Humbel, B.M. (2001). Enhanced resolution of membranes in cultured cells by cryoimmobilization and freeze-substitution. Microsc. Res. Tech. 53, 313-321. 